\title{
RESEARCH
}

\section{Exosomes increased angiogenesis in papillary thyroid cancer microenvironment}

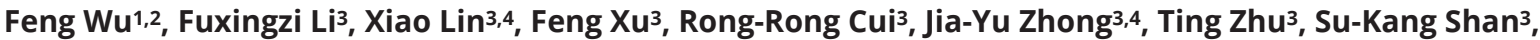 \\ Xiao-Bo Liao' ${ }^{5}$ Ling-Qing Yuan ${ }^{3}$ and Zhao-Hui Mo' ${ }^{1}$ \\ 1Department of Endocrinology, The Third Xiang-Ya Hospital, Central South University, Changsha, Hunan, People's Republic of China \\ 2Department of Pathology, The Second Xiang-Ya Hospital, Central South University, Changsha, Hunan, People's Republic of China \\ 3Department of Endocrinology and Metabolism, National Clinical Research Center for Metabolic Diseases, The Second Xiang-Ya Hospital, \\ Central South University, Changsha, Hunan, People's Republic of China \\ ${ }^{4}$ Department of Geriatrics, Institute of Aging and Geriatrics, The Second Xiang-Ya Hospital, Central South University, Changsha, Hunan, \\ People's Republic of China \\ ${ }^{5}$ Department of Cardiovascular Surgery, the Second Xiang-Ya Hospital, Central South University, Changsha, Hunan, People's Republic of China
}

Correspondence should be addressed to L-Q Yuan or Z-H Mo: allenylq@csu.edu.cn or easd04mzh@126.com

\begin{abstract}
Tumour-derived exosomes under hypoxic conditions contain informative miRNAs involved in the interaction of cancer and para-carcinoma cells, thus contributing to tissue remodelling of the tumour microenvironment (TME). Exosomes isolated from hypoxic papillary thyroid cancer cells, BCPAP cells and KTC-1 cells enhanced the angiogenesis of human umbilical vein endothelial cells (HUVECS) compared with exosomes isolated from normal thyroid follicular cell line (Nthy-ori-3-1), normoxic BCPAP or KTC-1 cells both in vitro and in vivo. miR-21-5p was significantly upregulated in exosomes from papillary thyroid cancer BCPAP cells under hypoxic conditions, while the exosomes isolated from hypoxic BCPAP cells with knockdown of miR-21-5p attenuated the promoting effect of angiogenesis. In addition, our experiment revealed that miR-21-5p directly targeted and suppressed TGFBI and COL4A1, thereby increasing endothelial tube formation. Furthermore, elevated levels of exosomal miR-21-5p are found in the sera of papillary thyroid cancer patients, which promote the angiogenesis of HUVECs. Taken together, our study reveals the cell interaction between hypoxic papillary thyroid cancer cells and endothelial cells, elucidating a new mechanism by which hypoxic papillary thyroid cancer cells increase angiogenesis via exosomal miR-21-5p/TGFBI and miR-21-5p/COL4A1 regulatory pathway.
\end{abstract}

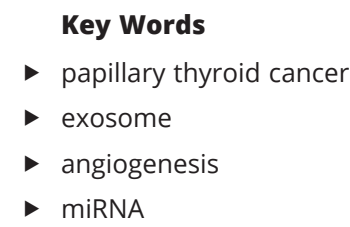

Endocrine-Related Cancer (2019) 26, 525-538

\section{Introduction}

Thyroid cancer is the most common endocrine malignancy, and its incidence is increasing in many countries of the world (Fagin et al. 2016). Among females, it is the fifth most common neoplasm after breast cancer, lung/bronchus cancer, colon/rectum and uterine corpus cancer (Jemal et al. 2008). Among the several histological subtypes of thyroid cancer, papillary thyroid cancer (PTC) is the most common pathological subtype, accounting for approximately $80-90 \%$ of all thyroid cancers (Aschebrook-Kilfoy et al. 2013). Although PTC is usually a well-differentiated thyroid cancer with a favourable prognosis, the clinical behaviour of PTC varies widely. Approximately 20\% of PTCs exhibit tumour recurrence, and some reach advanced stages. A recent survey reported
C) 2019 Society for Endocrinology Published by Bioscientifica Ltd. Printed in Great Britain 
an increasing trend with regard to mortality rates of thyroid cancer, mainly due to advanced stage PTC (Lim et al. 2017). Therefore, there is an urgent need to find the availability of markers of thyroid cancer aggressiveness and develop more efficient treatment options.

Growing evidence has revealed that the tumour microenvironment (TME), composed of the extracellular matrix and stromal cells, is a complex network, and cancer cells rely on the TME for sustained survival, growth, invasion and metastasis (Quail \& Joyce 2013, $\mathrm{Wu}$ et al. 2016). Increased vascular permeability in the tumour supports cancer growth and metastasis by continuously providing an adequate supply of oxygen and nutrients, thus increasing cancer cells' dissemination and premetastatic niche formation (Zhou et al. 2014). Hypoxia, an important element of the TME, has emerged as an important regulator of physiological and pathological processes. Under hypoxia, cancer cells secrete bioactive substances that modulate the TME to promote tumour angiogenesis. A threefold increase of angiogenic activity has been reported in PTC as compared with normal thyroid tissue, and increased angiogenic activity is associated with endothelial activation, increased capillary permeability and hyperperfusion (Akslen \& Livolsi 2000). Hypoxia-inducible factors (HIFs) are the vital mediators involved in the cellular adaptation to hypoxia. HIF-1, a key transcription factor of hypoxia, consists of an oxygenregulated HIF-1 $\alpha$ subunit and an aryl hydrocarbon nuclear translocator HIF-1 $\beta$ subunit (Pientka et al. 2012). Under normoxic conditions, HIF- $1 \alpha$ is hydroxylated and proteolysised by prolyl hydroxylases PHD1, PHD2 and PHD3, whereas under hypoxic conditions, the hydroxylases are no longer active, allowing HIF-1 $\alpha$ to translocate to the nucleus and regulate transcription (Triner \& Shah 2016).

Conventional signalling pathways responding to hypoxia include direct cell-cell contact or VEGF signalling, while previous studies have shown that exosomes, small endosome-derived vesicles, carry complex biological information consisting of proteins, mRNAs and miRNAs from hypoxic cancer cells diffusing to neighbouring cells or transported to distant locations where they can help to transduce signals or information in specific recipient cells (Tonini et al. 2003, Tadokoro et al. 2013). miRNAs are a class of 20-24 nt short non-coding RNAs that posttranscriptionally regulate gene expression (Lee et al. 2014). miR-21-5p is one of the most well-studied miRNAs and is implicated in promoting cancer growth and development that is often overexpressed in a wide variety of solid tumour tissues (Yao et al. 2009, Au Yeung et al. 2016). miR-21's pro-angiogenic function has been previously described and involves a synergistic effect with VEGF (Liu et al. 2016a). In addition, miR-21-5p has been found to increase angiogenesis by targeting multiple signalling pathways in various cancers, such as prostate cancer (Liu et al. 2011) and lung cancer (Zhao et al. 2013). However, how cancer cells adapt to hypoxia and communicate with the TME during PTC development remains largely unknown.

In this study, we investigate the potential role of exosomes in the hypoxia-dependent signalling between PTC cells and endothelial cells. Our research reveals that PTC cells and endothelial cells communicate through exosomal miR-21-5p released from PTC cells under hypoxia conditions, which may facilitate tumour angiogenesis without directly contacting contiguous tissue.

\section{Materials and methods}

\section{Ethics statement}

All experiments were reviewed and approved by the Ethics Committee of the Second Xiang-Ya Hospital, Central South University. The human study and human samples conformed to the principles outlined in the Declaration of Helsinki. All the animal and clinical human studies were formally approved by the Ethics Committee of the Second Xiang-Ya Hospital, Central South University. Written informed consent was obtained from all participants in our experiments.

\section{Cell lines and culture conditions}

Human PTC cells (BCPAP and KTC-1; Cell Bank of the Chinese Academy of Sciences, Shanghai, China) were cultured in RPMI medium 1640 (Gibco BRL) containing 10\% fetal bovine serum (FBS) (Gibco BRL), 1\% penicillin and streptomycin (PS) (Gibco BRL), 1\% nonessential amino acid (NEAA) (Invitrogen), 1\% GlutaMAX (Invitrogen) and $1 \%$ sodium pyruvate $100 \mathrm{mM}$ solution (Invitrogen). Human normal thyroid cell lines (Nthy-ori-3-1; CellBio, Shanghai, China) were cultured in high-glucose DMEM (Sigma) containing 10\% FBS and 1\% PS. Human umbilical vein endothelial cells (HUVECs) were obtained from the American Type Culture Collection. Cells were maintained at $37^{\circ} \mathrm{C}$ with $5 \% \mathrm{CO}_{2}$ in a humidified environment.
C) 2019 Society for Endocrinology Published by Bioscientifica Ltd. Printed in Great Britain 


\section{Cell proliferation assay}

Cell proliferation was monitored using CCK-8 assay (7-Sea Biotech, Shanghai, China) following the manufacturer's protocol. Briefly, $2 \times 10^{3}$ cells per well were allowed to grow in 96-well plates, and were treated with exosomes that were derived from Nthy-ori-3-1 and BCPAP cells under normoxic or hypoxic conditions for Days 1, 2, 3 and 4 . Ten microliters of CCK- 8 reagent were added to each well at $4 \mathrm{~h}$ before the endpoint of incubation. The optical density (OD) $450 \mathrm{~nm}$ values were determined by a microplate reader (NanoDrop 2000). All experiments were repeated at least three times.

\section{Isolation of exosomes from cell supernatants and patient sera}

When BCPAP, KTC-1 and Nthy-ori-3-1 cells reached $80 \%$ confluence, the cells were washed with PBS and cultured under hypoxic $\left(1 \% \mathrm{O}_{2}\right)$ or normoxic $\left(20 \% \mathrm{O}_{2}\right)$ conditions in cell culture medium containing exosomefree FBS (prepared by overnight ultracentrifugation at $100,000 \mathrm{~g}$ at $4^{\circ} \mathrm{C}$ ) for $24 \mathrm{~h}$. the medium was centrifuged at $500 \boldsymbol{g}$ for $15 \mathrm{~min}$ and $10,000 \boldsymbol{g}$ for $20 \mathrm{~min}$ to remove dead cells and cellular debris. Exosomes were isolated by ultracentrifugation at $100,000 \boldsymbol{g}$ for $70 \mathrm{~min}$ and washed in PBS using the same ultracentrifugation conditions. Then, the supernatant was removed by aspiration. The exosome pellets were resuspended in $200 \mu \mathrm{L}$ PBS. All procedures were performed at $4^{\circ} \mathrm{C}$.

Pre-operative blood samples were obtained from 20 PTC patients with lymph node metastasis and 20 donors admitted to the division of breast and thyroid surgery at the Second Xiang-Ya Hospital, Central South University, Hunan, China. These patients were diagnosed with PTC according to WHO classification by two pathologists. And 20 donors were diagnosed with benign thyroid nodule by two pathologists. The clinical data and information corresponding to the specimens are shown in Supplementary Table 2 (see section on supplementary data given at the end of this article). Sera was separated by centrifugation and frozen at $-80^{\circ} \mathrm{C}$. The sera was centrifuged at $300 \boldsymbol{g}$ for $10 \mathrm{~min}$ and $2000 \boldsymbol{g}$ for 10 min to remove cellular debris. Then, the supernatant was centrifuged at $10,000 \boldsymbol{g}$ for $30 \mathrm{~min}$ followed by ultracentrifugation for $70 \mathrm{~min}$ at $100,000 \mathrm{~g}$. The pelleted exosomes were washed twice with a large volume of PBS and centrifuged at $100,000 \boldsymbol{g}$ for $70 \mathrm{~min}$, and then resuspended in $15 \mathrm{~mL}$ of PBS. The exosome suspension was filtered through a $0.22 \mu \mathrm{m}$ filter (Millipore) and centrifuged at $4000 \mathrm{~g}$ to about $200 \mu \mathrm{L}$ by ultra-filtration in a $15 \mathrm{~mL}$ Amicon Ultra-15 Centrifugal Filter Unit (100 kDa; Millipore). All procedures were performed at $4^{\circ} \mathrm{C}$. Exosomes were stored at $-80^{\circ} \mathrm{C}$ or used for the downstream experiments. Exosome protein content was quantified using the bicinchoninic acid protein assay (Chen et al. 2018).

\section{Plasmid constructs and luciferase reporter assay}

Full details are given in the Supplementary Materials and methods (see section on supplementary data given at the end of this article). Briefly, wild-type and mutant segments of TGFBI and COL4A1 3-'UTR, including predicted miR-21-5p binding sites, were cloned into luciferase reporter vector pmirGLO (Promega) PmeI and XbaI restriction sites. Mutant TGFBI and COL4A1 3-'UTR were produced using the QuikChange Site-Directed Mutagenesis Kit (Stratagene). HUVECs were co-transfected with a luciferase reporter carrying wild-type or mutant TGFBI and COL4A1 3-'UTR, and miR-21-5p mimics or mimic control. After transfection, luciferase activities were detected with the luciferase assay system (Promega). PCR and mutagenic primers are given in Supplementary Table 1.

\section{Tube formation assay, scratch wound assay, cell migration assay}

Full details are given in the Supplementary Materials and methods. Briefly, growth factor-reduced Matrigel (BD Biosciences) was plated in pre-cooled 24-well plates and incubated at $37^{\circ} \mathrm{C}$. HUVECs were treated with exosomes isolated from a supernatant of Nthy-ori-3-1 cells, BCPAP cells under hypoxic conditions or BCPAP cells under normoxic conditions for $24 \mathrm{~h}$ and then plated on reduced growth factor Matrigel in 24-well plates for $6 \mathrm{~h}$. The total branching points were quantified by ImageJ software (NIH).

\section{Gene expression estimated using qRT-PCR}

Total RNA was extracted from the cells using TRIzol reagent (Invitrogen) and from exosomes using miRNeasy Micro Kit (Qiagen), and cDNA was prepared using an All-in-One first-strand cDNA synthesis kit (catalogue no. AORT-0060, Genecopoeia) as previously described (Cui et al. 2012, Liao et al. 2013). For analysis of miRNA expression, quantitative reverse transcription-polymerase chain reaction (qRT-PCR) (Genecopoiea, MmiRQP0538, Guangzhou, China) was carried out using the primers https://erc bioscientifica com https://doi.org/10.1530/ERC-19-0008 (c) 2019 Society for Endocrinology Published by Bioscientifica Ltd. Printed in Great Britain 
for human miR-21-3p (catalogue no. HmiRQP0315), miR-21-5p (catalogue no. HmiRQP0316), miR-155-3p (catalogue no. HmiRQP0220), miR-155-5p (catalogue no. HmiRQP0221), miR-181a-3p (catalogue no. HmiRQP0231), miR-181a-5p (catalogue no. HmiRQP0232), miR-221-3p (catalogue no. HmiRQP0338), miR-221-5p (catalogue no. HmiRQP0337), miR-222-3p (catalogue no. HmiRQP0339), miR-222-5p (catalogue no. HmiRQP0340), miR-16-5p (catalogue no. HmiRQP0227) and U6 snRNA (catalogue no. HmiRQP9071) (Genecopoiea), according to the manufacturer's instructions. Briefly, $20 \mu \mathrm{L}$ reactions were incubated in a 96 -well optical plate at $95^{\circ} \mathrm{C}$ for $10 \mathrm{~min}$, followed by 40 cycles of $95^{\circ} \mathrm{C}$ for $10 \mathrm{~s}, 62^{\circ} \mathrm{C}$ for $20 \mathrm{~s}$ and $72^{\circ} \mathrm{C}$ for $10 \mathrm{~s}$. Cellular miRNA data were normalised to U6, and exosmal miRNA data were normalised to miR-16-5p. Relative quantification was calculated through the $2^{-\Delta \Delta C T}$ method.

\section{Western blot analysis}

Western blot analysis was carried out for the detection of CD63, CD81, TSG101, HIF-1 $\alpha$, COL4A1, TGFBI, GAPDH or $\beta$-actin protein levels as previously described (Yuan et al. 2010, Liao et al. 2013, Liu et al. 2016b, Lin et al. 2018). Briefly, $30 \mu \mathrm{L}$ exosome lysate or $30 \mu \mathrm{g}$ of protein from each cell layer extract was loaded onto the same SDS PAGE and then transferred to a PVDF membrane. After blocking with 5\% non-fat milk, the membrane was incubated with primary antibodies overnight at $4^{\circ} \mathrm{C}$. The primary antibodies include anti-CD63 (1:200; Santa Cruz Biotechnology), anti-CD81 (1:200; Santa Cruz Biotechnology), anti-TSG101 (1:200; Santa Cruz Biotechnology), anti-HIF-1 $\alpha$ (1:500; Novus, Centennial, CO, USA), anti-COL4A1 (1:1000; GeneTex, Irvine, CA, USA), anti-TGFBI (1:250; Novus), anti-GAPDH (1:5000; Abgent, San Diego, CA, USA) and anti- $\beta$-actin (1:5000; Abgent). The next morning, the membrane was washed with PBS three times every $10 \mathrm{~min}$. The membrane was then incubated with the HRP-conjugated goat-anti-rabbit (1:5000; Santa Cruz Biotechnology) or HRP-conjugated goat-anti-mouse (1:5000; Santa Cruz Biotechnology) antibody in $2 \%$ non-fat milk for $1 \mathrm{~h}$. Blots were processed using an ECL kit, exposed to film and then analysed by densitometry.

\section{In vivo Matrigel plug assay}

Equal amount of exosomes derived from BCPAP cells cultured under normoxic or hypoxic conditions or BCPAP cells treated with a miR-21-5p inhibitor under hypoxic conditions or Nthy-ori-3-1 cells were mixed with $200 \mu \mathrm{L}$ of growth factor reduced Matrigel. The mixture solution was subcutaneously injected into nude mice (males, 8-week-old BALB/c, $n=3$ ). After 13 days, the Matrigel plugs were harvested and dissected, and then analysed by immunohistochemical analysis. All animal care was in accordance with institutional guidelines.

\section{Immunohistochemical staining}

Immunohistochemical staining for CD31, TGFBI and COL4A1 was performed as previously described (Wang et al. 2017). TGFBI and COL4A1 expression was performed on PTC tissues and on histologically normal thyroids. The normal thyroid tissues were obtained from surgery for benign thyroid nodule. Samples were fixed and processed by the paraffin-embedded method. Tissue sections were deparaffinised in xylene and rehydrated in a graded ethanol series. To clear endogenous peroxidase, sections were incubated with 3\% hydrogen peroxide, followed by antigen retrieval with trypsin. After blocking with $5 \%$ BSA, slides were probed overnight at $4^{\circ} \mathrm{C}$ with antibodies against CD31 (1:300; Servicebio, Wuhan, China), TGFBI (1:100; Novus) and COL4A1 (1:100; GeneTex). The primary antibody was detected by a biotinylated secondary antibody followed by the avidinbiotin peroxidase complex and 3,3'-diaminobenzidine chromogen (catalogue no. GK500710; GeneTech, Shanghai, China). Slides were counterstained with haematoxylin and images were taken with a light microscope (Olympus). Stained slides were scored according to the intensity of staining $(-: 0 ;+: 1 ;++: 2$ and $+++: 3)$ and percentage of the endothelial cells staining positive for each antigen (0\%: 0; 1 29\%: 1; 30 69\%: 2; 70 100\%: 3). The intensity score was multiplied by the percentage score to obtain a final score used in the statistical analyses (Zhou et al. 2014).

\section{Statistical analysis}

The results of the experiments are presented as means \pm s.D., and analysis was performed with Statistical Product and Service Solutions (SPSS) software (version 19.0). Differences between groups were evaluated by an analysis of variance (one-way ANOVA) or Student's $t$-test. $P<0.05$ was considered statistically significant. All experiments were repeated at least three times, and representative experiments are shown in the figures.
(C) 2019 Society for Endocrinology Published by Bioscientifica Ltd. Printed in Great Britain 


\section{Results}

\section{Isolation and characterisation of exosomes derived from PTC cells under normoxic or hypoxic conditions}

We isolated and characterised exosomes from Nthyori-3-1 cells, BCPAP cells under normoxic conditions and BCPAP cells under hypoxic conditions for $24 \mathrm{~h}$ using TEM and immunoblot (using CD81, CD63 and TSG101). TEM revealed that the size of exosomes was similar among the normoxic Nthy-ori-3-1, normoxic BCPAP and hypoxic BCPAP cells, and each vesicle showed the classic cup-shaped appearance (Fig. 1A). Exosomal markers, including CD63, CD81 and TSG101, were also observed in exosomes isolated from the Nthy-ori-3-1, normoxic BCPAP and hypoxic BCPAP cells (Fig. 1B). Nano-vesicles with sizes $30-100 \mathrm{~nm}$ were found in all the exosomes isolated from the Nthy-ori-3-1, normoxic BCPAP and hypoxic BCPAP cells (Fig. 1C). All these data confirmed these nanoparticles were exosomes. Compared to BCPAP cells cultured in normoxia, the expression of HIF- $1 \alpha$ in BCPAP cells increased under $1 \% \mathrm{O}_{2}$ concentrations for $24 \mathrm{~h}$ (Fig. 1D), which suggested the successful establishment of a hypoxic environment.

\section{Exosomes derived from hypoxic cancer cells induce angiogenic activities in HUVECs}

Both tumour and endothelial cells are in hypoxic environments in situ, particularly when vascular endothelial cells are close to cancer cells. To study the effect of exosomes derived from hypoxic thyroid cancer cells on endothelial cells, we added the exosomes derived from Nthy-ori-3-1 or BCPAP cells with or without hypoxic environment to HUVECs and determined the exosome's biological functions on HUVECS. It was shown that the exosomes derived from hypoxic BCPAP cells increased tube formation (Fig. 2A and B) and migration determined by transwell assay (Fig. 2C and D) and scratch wound assay (Fig. 2E and F) of HUVECs compared with the exosomes derived from normoxic BCPAP and Nthy-ori-3-1 cells. Moreover, similar trends of angiogenic activities were also observed in another PTC cell line KTC-1. The exosomes derived from hypoxic KTC-1 cells increased tube formation (Supplementary Fig. 1A and B), migration determined by transwell assay (Supplementary Fig. 1C and D) and scratch wound assay (Supplementary Fig. 1E and F) of HUVECs compared with the exosomes derived from normoxic KTC-1 and Nthy-ori-3-1 cells. The CCK-8 assay showed that proliferation is increased significantly in HUVECs treated with exosomes from hypoxic BCPAP cells, compared with that treated with exosomes from normoxic BCPAP and Nthy-ori-3-1 cells (Fig. 2G). To analyse the angiogenic responses to exosomes derived from hypoxic BCPAP cells in vivo, we performed a Matrigel plug angiogenesis assay to detect the newly formed blood vessels in the transplanted gel plugs in nude mice. The density of the neovessel lines in Matrigel plugs was quantified by immunohistochemical staining with antimouse CD31. The plugs containing the exosomes derived from hypoxic BCPAP cells significantly increased the density of CD31+ neovessels compared with the exosomes derived from normoxic BCPAP and Nthy-ori-3-1 cells
A

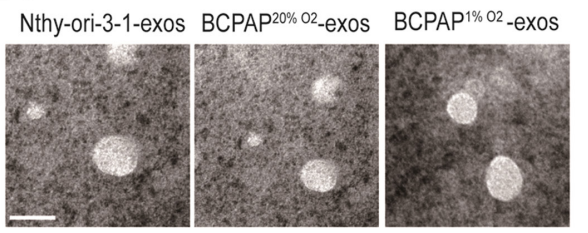

Size Distribution by Intensity

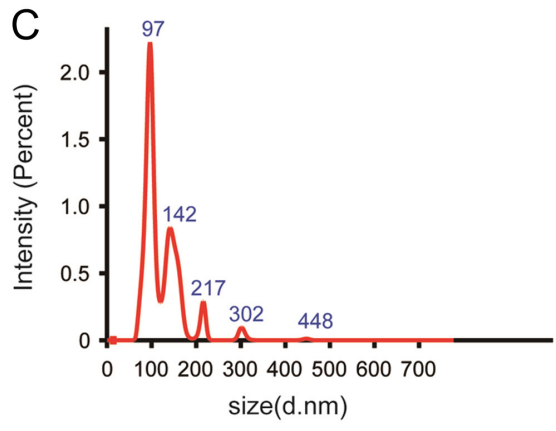

B

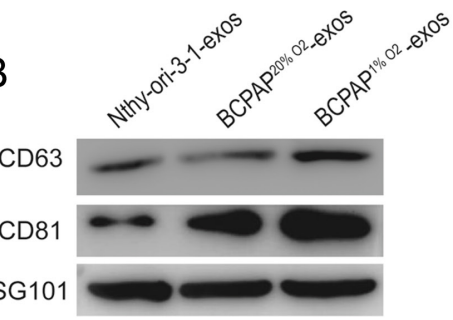

D

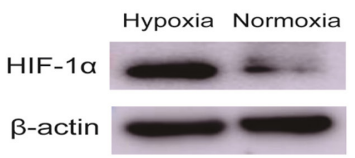

Figure 1

Characterisation of exosomes derived from cell culture medium. (A) Transmission electron micrographs of exosomes derived from Nthy-ori-3-1 or BCPAP cells cultured under 20 or $1 \% \mathrm{O}_{2}$ conditions. Bar $=100 \mu \mathrm{m}$. (B) CD63, CD81 and TSG101 immunoblots of exosomes derived from Nthy-ori-3-1 or BCPAP cells cultured under 20 or $1 \% \mathrm{O}_{2}$ conditions. These exosomes were isolated from $15 \mathrm{~mL}$ of culture media of Nthy-ori-3-1 or BCPAP cells cultured under 20 or $1 \% \mathrm{O}_{2}$ conditions for $24 \mathrm{~h}$, respectively. The same amounts of exosome lysate $(30 \mu \mathrm{L})$ were loaded in each lane of the gels. (C) The nanoparticle concentration and size distribution of the exosomes derived from culture media of BCPAP cells cultured under $1 \% \mathrm{O}_{2}$ conditions for $24 \mathrm{~h}$. (D) The expression of HIF- $1 \alpha$ in BCPAP cells under normoxic and hypoxic conditions for $24 \mathrm{~h}$. 
A

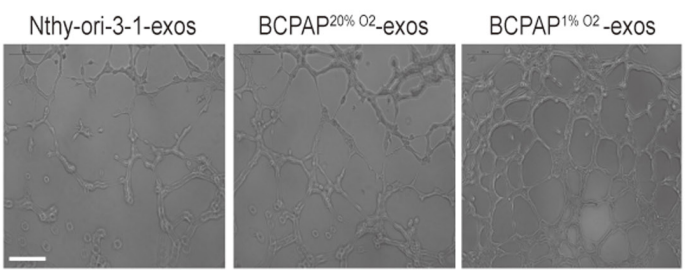

C

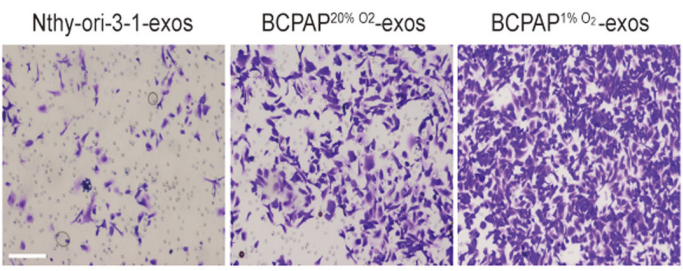

E

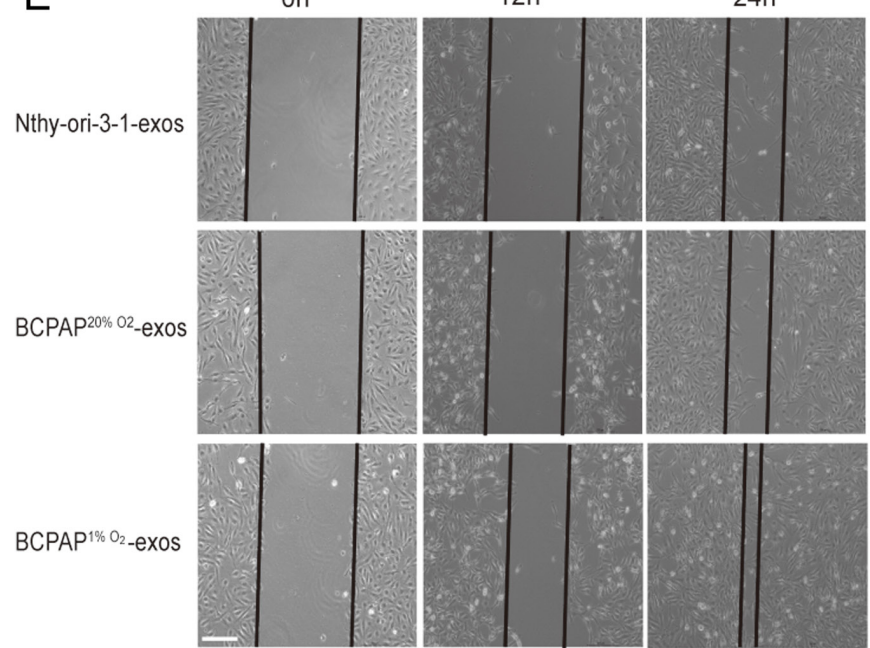

\section{G}

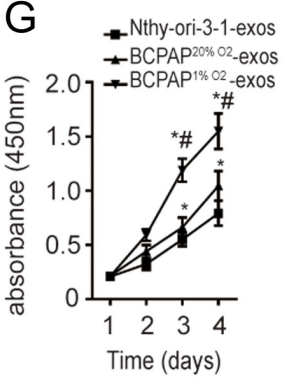

$\mathrm{H}$
B
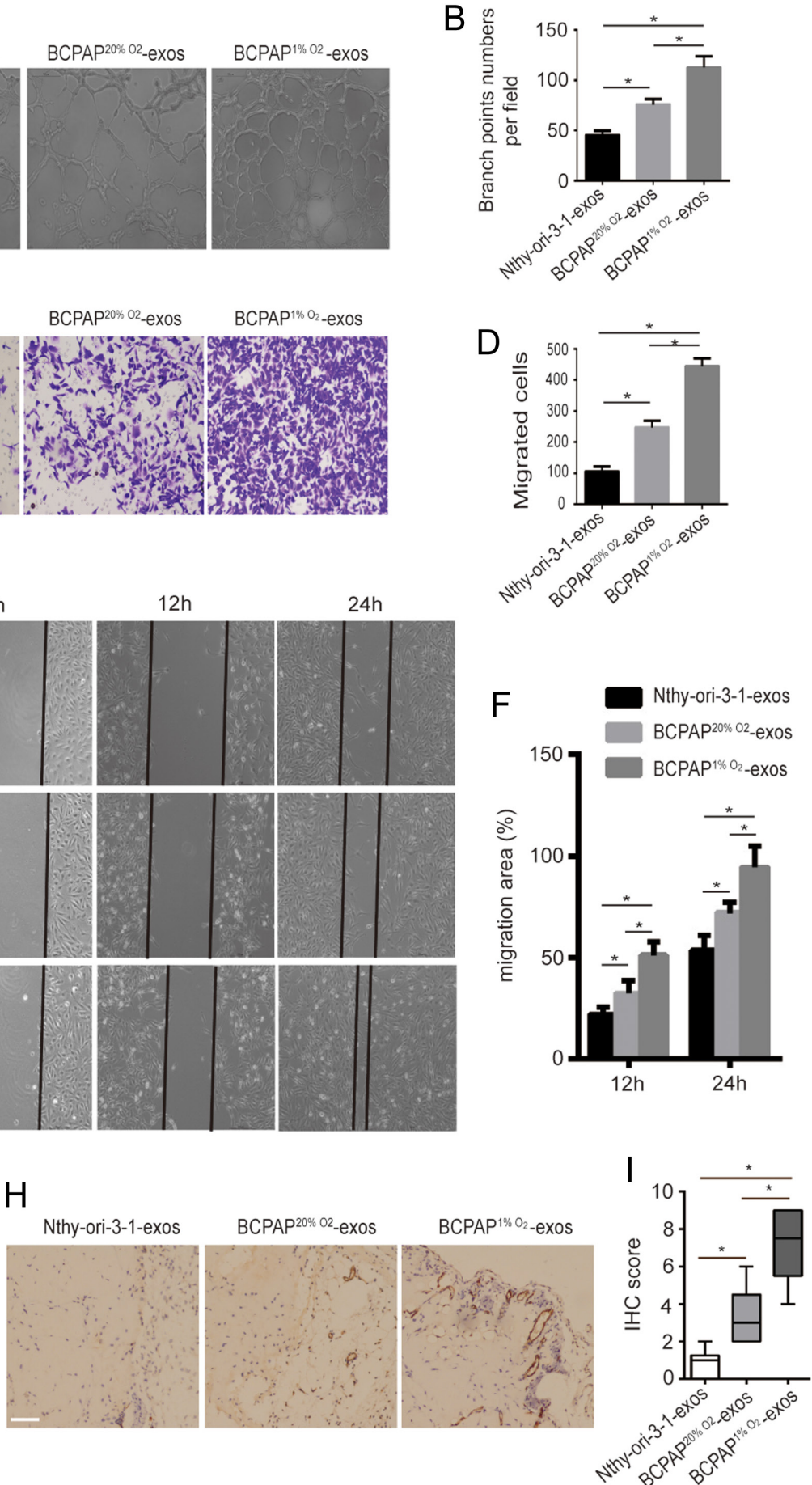

Figure 2

Exosomes derived from hypoxic BCPAP cells induce angiogenic activities in HUVECs. (A) Endothelial tube formation of HUVECs cultured with Nthy-ori-3-1exos, BCPAP20\%02-exos or BCPAP1\%02-exos for $6 \mathrm{~h}$. (B) Quantitative analysis of the total branching points in (A). $n=3$ per group. (C) BCPAP1\%02-exos promoted migration of HUVECs. HUVECs were plated in the upper chamber and then incubated with exosomes ( $20 \mu \mathrm{g} / \mathrm{mL})$ for $24 \mathrm{~h}$. Photomicrographs depict the migrated HUVECs at the end of the experiment. (D) The number of migrated cells was counted, and the results were presented as mean \pm S.D., from three individual experiments. (E) An artificial wound was created on the monolayer of HUVECS at time zero (T = $0 \mathrm{~h}$ ). After capturing of the denuded area in each well, cells were incubated with indicated concentrations of exosomes for $24 \mathrm{~h}$; the denuded area was then captured at $12 \mathrm{~h}$ and $24 \mathrm{~h}(\mathrm{~F})$, and the percentages of wound recovery were calculated. Data were presented as mean \pm S.D. from triplicate experiments. (G) Proliferation of HUVECS cultured with Nthy-ori-3-1-exos, BCPAP20\%02-exos or BCPAP1\%02-exos were assessed by CCK-8 assays. (H) The neovessels induced by the exosomes in Matrigel were visualised by immunohistochemical staining with anti-mouse CD31 antibody. (I) IHC score in (H) were analysed. Data were presented as mean \pm S.D. from triplicate experiments. ${ }^{*} P<0.05$ compared with Nthy-ori-3-1-exos, $\# P<0.05$ compared with $\mathrm{BCPAP}_{20} \% \mathrm{O}_{2}$-exos. $\mathrm{Bars}=200 \mu \mathrm{m}$. 
(Fig. 2H and I). These experiments demonstrated that the exosomes from hypoxic thyroid cancer cells enhanced the angiogenic activities of HUVECs both in vitro and in vivo.

\section{miR-21-5p is secreted by hypoxic thyroid cancer cells and transferred to endothelial cells via exosome secretion}

To confirm the exosome secreted by thyroid cells can be uptaken by endothelial cells, we added the PKH26-labelled exosomes of Nthy-ori-3-1 and BCPAP cells to HUVECs. Figure 3A shows that the HUVECs exhibited an efficient uptake of exosomes secreted by these cells. To identify the exosome-associated functional molecules that mediated the pro-angiogenic effects of hypoxic BCPAP cells-derived exosomes, qRT-PCR analysis was performed to detect the expression levels of a class of miRNAs (including miR-21, miR-34a, miR-155, miR-181a, miR-221 and miR-222) that have positive roles in regulating tumour angiogenesis (Kong et al. 2014, Lee et al. 2014, Yang et al. 2015, Liu et al. 2016a, Bayraktar et al. 2018, Sun et al. 2018) (Fig. 3B, C and Supplementary Fig. 2). As shown in Fig. 3B and C, levels of miR-21-5p and miR-221-5p increased in BCPAP cell-derived exosomes under hypoxic conditions when compared with exosomes derived from Nthy-ori-3-1 and BCPAP cells under normoxic conditions. Of these, miR-21-5p was the most highly expressed miRNA among the detected miRNAs in hypoxic BCPAP cell-derived exosomes (Fig. 3D). Studies have indicated that miR-21 can promote the angiogenic activities of endothelial cells (Liu et al. 2016a); thus, we focused on exosomal miR-21-5p for further investigation.
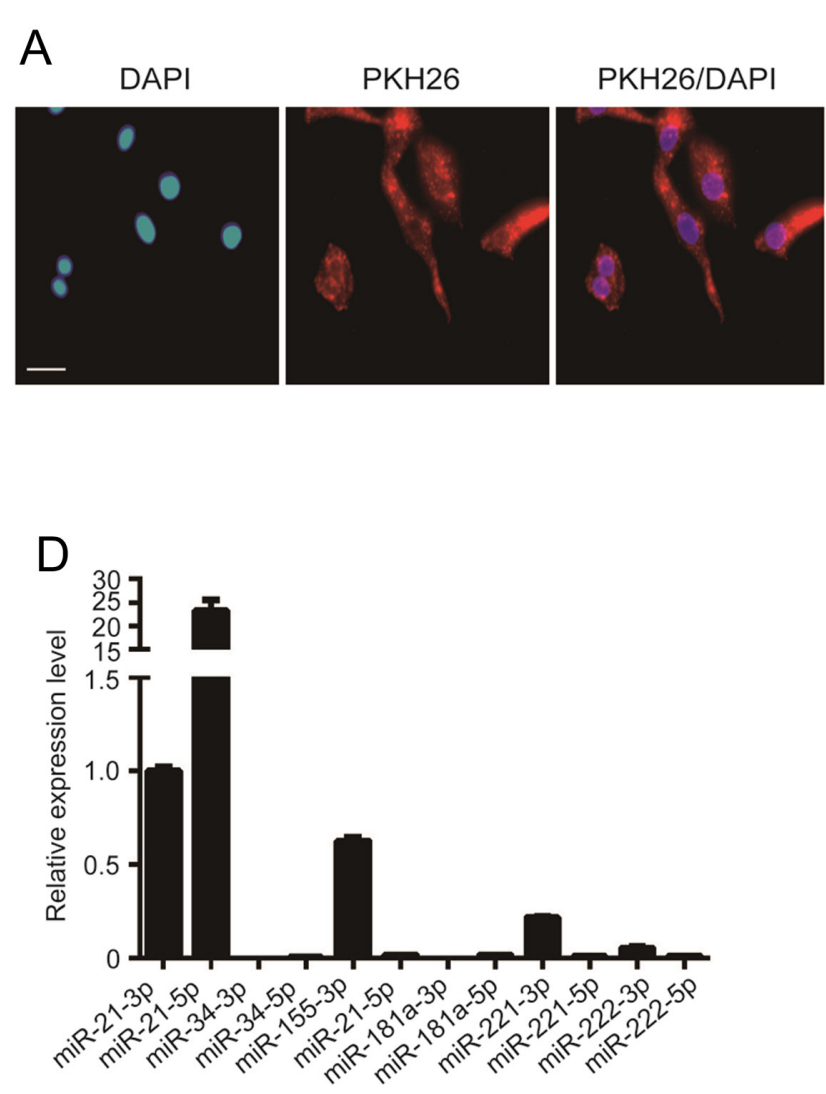
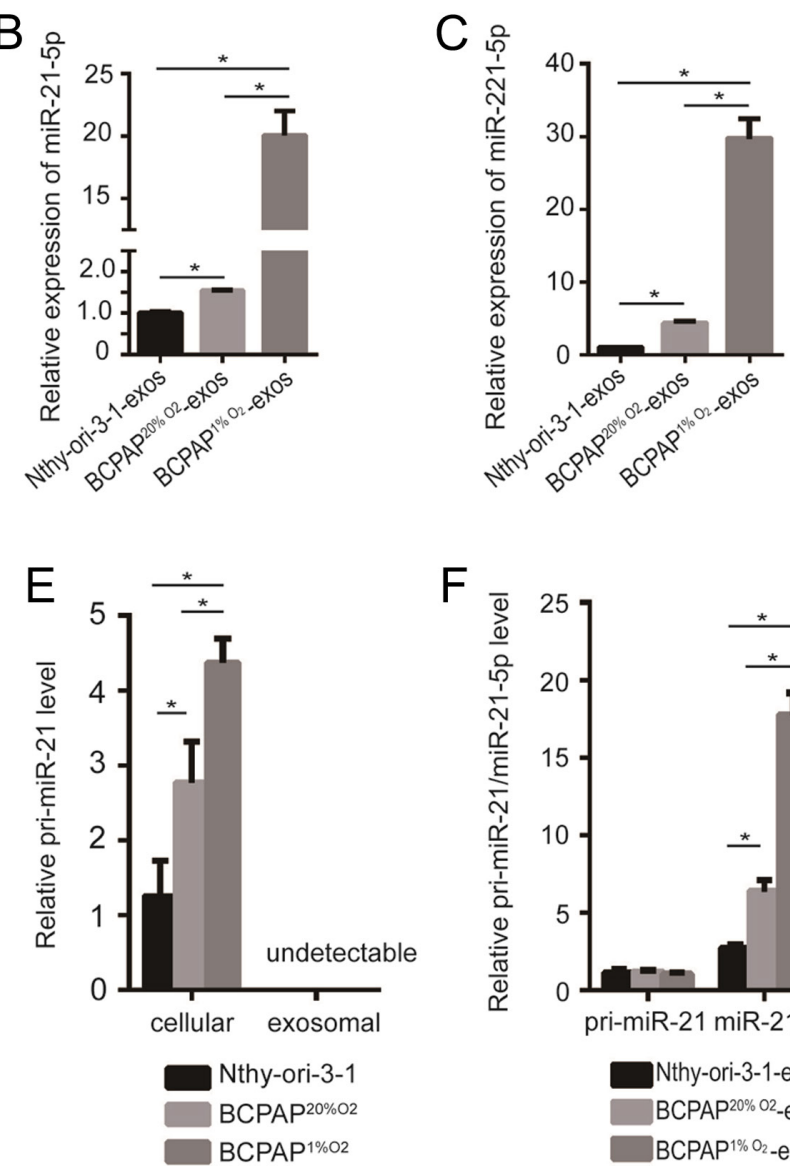

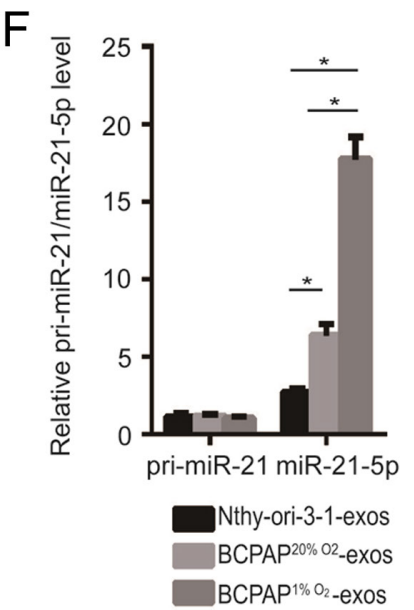

Figure 3

Exosomes deliver miR-21-5p into HUVECs. (A) Fluorescence microscopy analysis of PKH26-labelled exosome internalisation by HUVECs. The red-labelled exosomes were visible in the cytoplasmic region of recipient cells. Bar $=20 \mu \mathrm{m}$. (B and C) HUVECs incubated with BCPAP1\%02-exos for $3 \mathrm{~h}$ showed higher expression levels of miR-21-5p and miR-221-5p than those incubated with Nthy-ori-3-1-exos and BCPAP20\%02-exos. $n=3$ per group. (D) Detection of the expression of the indicated miRNAs by qRT-PCR analysis in BCPAP1\%02-exos. $n=3$. (E) Cellular and exosomal RNA of Nthy-ori-3-1, BCPAP20\%02 and BCPAP $1 \% 02$ were subjected to RT-qPCR for pri-miR-21-5p. (F) Expression levels of pri-miR-21-5p and mature miR-21-5p in HUVECs incubated with Nthy-ori-3-1-exos, BCPAP20\%02-exos or BCPAP1\%02-exos. Data are presented as mean \pm s.D. from triplicate experiments. ${ }^{*} P<0.05$ compared with Nthy-ori-3-1 group. 
To further confirm that hypoxic BCPAP cells secreted miR-21-5p can be transferred to endothelial cells via exosomes, we determined the primary(pri)-miR-21 and mature miR-21-5p levels in exosomes and HUVECs treated with the exosomes isolated from Nthy-ori-3-1, normoxic BCPAP and hypoxic BCPAP cells. We found that pri-miR-21 only exhibited higher intracellular levels in Nthy-ori-3-1 and BCPAP cells, while it was not detectable in exosomes (Fig. 3E). Furthermore, an increase in cellular levels of mature miR-21-5p, but not pri-miR-21, was observed in recipient HUVECs following their treatment with exosomes of hypoxic BCPAP cells (Fig. 3F). These data suggested that the exosomes of hypoxic BCPAP cells containing mature miR-21-5p were internalised by vascular endothelial cells.

\section{Depletion of miR-21-5p reduces hypoxic BCPAP cell-derived exosome functional effects in HUVECs}

To investigate whether HUVECs were affected by exosomal miR-21-5p, we reduced miR-21-5p levels by transfecting BCPAP cells with miR-21-5p inhibitors (anti-miR-21-5p). As determined by qRT-PCR, these transfected anti-miR-21-5p BCPAP cells produced exosomes with reduced miR-21-5p levels (Fig. 4A). The exosomes released from anti-miR-21-5p-transfected BCPAP cells significantly reduced the tube formation and migration ability of HUVECs compared to non-transfected BCPAP cells (Fig. 3B, C, D and E). Furthermore, the plugs containing the exosomes derived from anti-miR-21-5p-transfected BCPAP $1 \% \mathrm{O}_{2}$ cells significantly reduced the density of $\mathrm{CD} 1^{+}$neovessels when compared with the exosomes derived from non-transfected hypoxic BCPAP cells (Fig. $4 \mathrm{~F}$ and $\mathrm{G}$ ). These data suggested that the depletion of miR-21-5p reduces the tube formation and migration ability of HUVECs.

\section{Exosomal miR-21-5p derived from hypoxic PTC cells targets TGFBI and COL4A1}

We predicted the possibility of miR-21-5p targets that contribute to the angiogenic function using

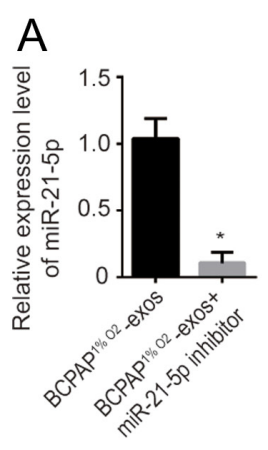

B
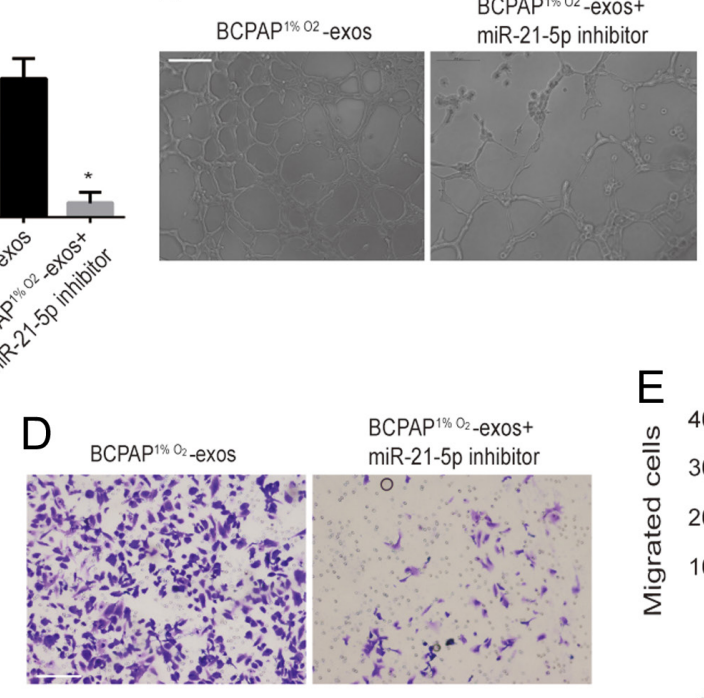

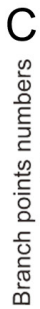
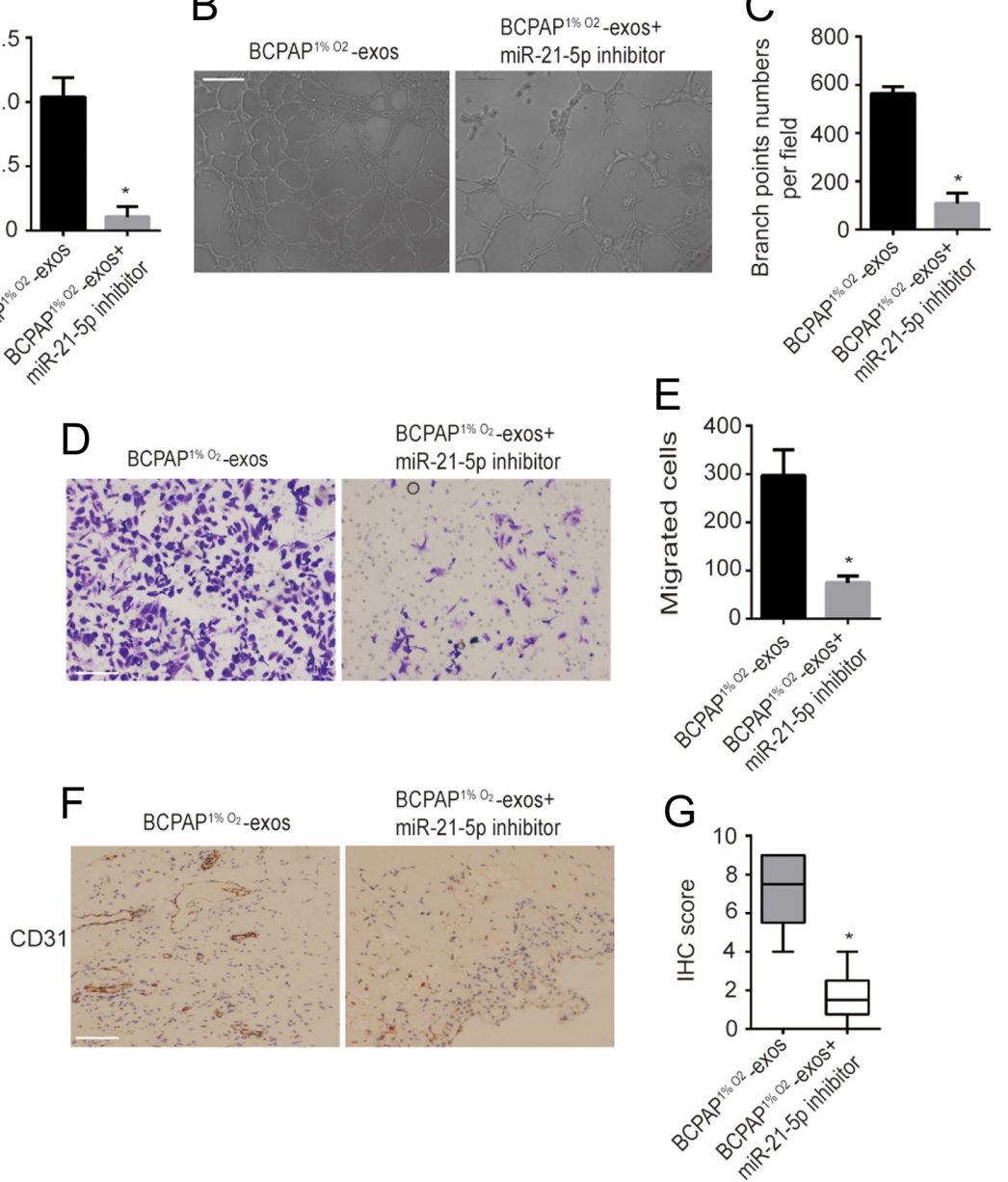

\section{Figure 4}

Depletion of miR-21-5p reduces hypoxic BCPAP cells-derived exosome functional effects in HUVECs. (A) qPCR analysis of miR-21-5p in exosomes of miR-21-5p inhibitors transfected hypoxic BCPAP cells and non-transfected hypoxic BCPAP cells. (B) Endothelial tube formation of HUVECS cultured with BCPAP $1 \% 02$-exos or miR-21-5p inhibitors transfected BCPAP1\%02-exos for $6 \mathrm{~h}$. (C) Quantitative analysis of the total branching points in (B). $n=3$ per group. (D) HUVECs were plated in the upper chamber and then incubated with exosomes $(20 \mu \mathrm{g} / \mathrm{mL})$ for $24 \mathrm{~h}$. Photomicrographs depict the migrated HUVECS at the end of the experiment. (E) The number of migrated cells was counted; the results were presented as mean \pm S.D. from three individual experiments. ( $F$ ) The neovessels induced by exosomes in Matrigel were visualised by immunohistochemical staining with antimouse CD31 antibody. (G) IHC score in (F) were analysed. Data were presented as mean \pm S.D. from triplicate experiments. ${ }^{*} P<0.05$ compared with BCPAP $1 \% 02$-exos. Bars $=200 \mu \mathrm{m}$. 
online database (TargetScan). Analysis revealed TGFBI and COL4A1 may be the possible targets of miR-21-5p (Fig. 5A and $\mathrm{B})$. To confirm the direct binding between miR-21-5p and the TGFBI 3 'UTR regions, we conducted a reporter assay using the luciferase reporter plasmid containing WT and mutation (MT) TGFBI 3'UTR with the miR-21-5p-binding site. miR-21-5p mimics transfection reduced luciferase activity of reporter constructs containing the WT-TGFBI 3'-UTR, but not the MUT-TGFBI 3'-UTR. The mimics' control did not affect the WT or mutant constructs, confirming the specificity of action (Fig. 5C). Similarly, to confirm the direct binding between miR-21-5p and the COL4A1 3'UTR regions, we conducted a reporter assay using the luciferase reporter plasmid containing WT and mutation (MT) COL4A1 3'UTR with the miR-21-5p-binding site. miR-21-5p mimics transfection reduced luciferase activity of reporter constructs containing the WT-COL4A 3'-UTR, but not the MUT-COL4A 3'-UTR. The mimics control did not affect the WT or mutant constructs, confirming the specificity of action (Fig. 5D). Consistent with the results from the luciferase reporter assay, treatment with exosomes derived from hypoxic BCPAP cells resulted in a significant decrease in TGFBI and COL4A1 expression in HUVECs (Fig. 5E). Similarly, transfection with miR-21-5p mimics significantly decreased TGFBI and COL4A1 expression in HUVECs, while transfection with miR-21-5p inhibitors resulted in a significant increase of TGFBI and COL4A1 expression in HUVECs (Fig. 5F). These findings indicated that exosomal miR-21-5p of PTC under hypoxic conditions inhibits TGFBI and COL4A1 expression and increases angiogenesis in recipient vascular endothelial cells.

\section{Inhibition of TGFBI or COL4A1 induces pro-angiogenic effects on HUVECs}

We next asked whether knocking down the expression of miR-21-5p target gene TGFBI or COL4A1 could achieve positive effects on HUVEC function similar to BCPAP cellsderived exosomes under hypoxic conditions. Three TGFBI siRNAs (siTGFBI \#1, 2 and 3) were used to downregulate the expression of TGFBI in HUVECs. The inhibitory
A

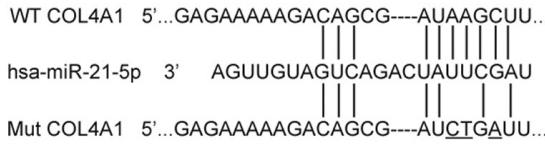

B

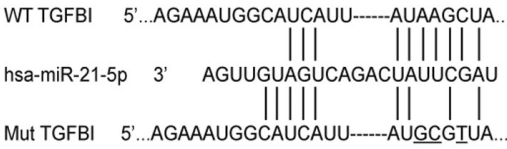

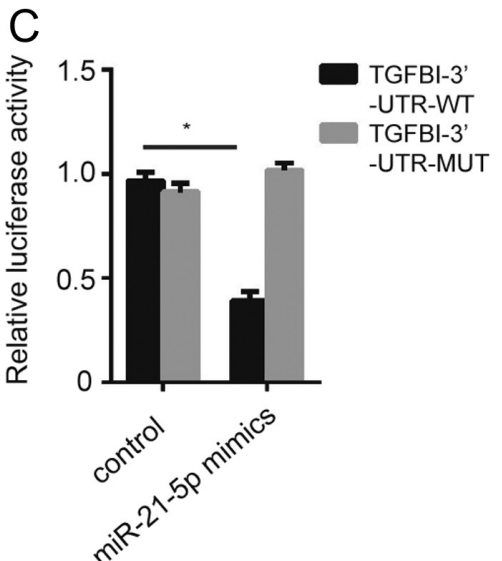

E

COL4A1

TGFBI

$\beta$-actin
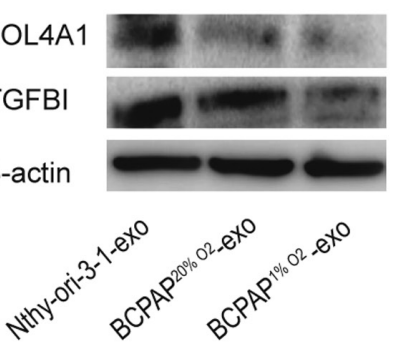

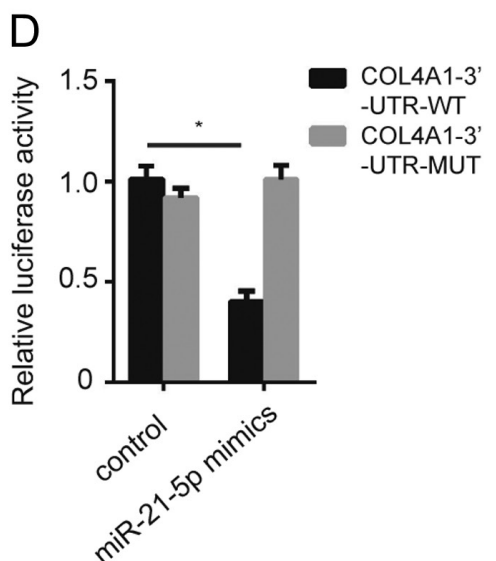

F
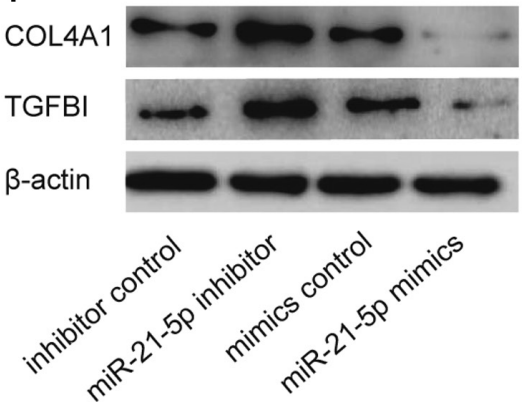

Figure 5

miR-21-5p targets the $3^{\prime} U T R$ of the TGFBI and COL4A1 genes in HUVECS. (A) Schematic of the miR-21-5p putative target site in human TGFBI $3^{\prime}$ UTR. (B) Schematic of the miR-21-5p putative target site in human COL4A1 3'UTR. (C) HUVECS were co-transfected with the luciferase reporter carrying WT-TGFBI-3'UTR or MUT-TGFBI-3'UTR and miR-21-5p or control miR mimics. $48 \mathrm{~h}$ after transfection, Firefly luciferase values, normalised for Renilla luciferase, are presented. (D) HUVECS were co-transfected with the luciferase reporter carrying WT-COL4A1-3'UTR or MUT-COL4A1-3'UTR and miR-21-5p or control miR mimics. Forty-eight hours after transfection, Firefly luciferase values, normalised for Renilla luciferase, are presented. (E) TGFBI and COL4A1 protein levels by Western blot analysis ( $30 \mu \mathrm{g}$ of protein were used for each Western blot performed) were tested $48 \mathrm{~h}$ after exosomes were treated. (F) TGFBI and COL4A1 protein levels by Western blot analysis ( $30 \mu \mathrm{g}$ of protein were used for each Western blot performed) were tested $48 \mathrm{~h}$ after HUVECs were transfected with miR-21-5p mimics control, miR-21-5p mimics, miR-21-5p inhibitor control or miR-21-5p inhibitors. ${ }^{*} P<0.05$ compared with the control group. 
efficiency of these three siRNAs was verified by Western blotting, and the most effective siRNAs were used for the following functional assays (Fig. 6A). Similarly, three COL4A1 siRNAs (siCOL4A1 \#1, 2 and 3) were used to downregulate the expression of COL4A1 in HUVECs. The inhibitory efficiency of these three siRNAs was verified by Western blotting, and the most effective siRNAs were used for the following functional assays (Fig. 6B). The results of tube formation and migration assay revealed that the inhibition of TGFBI or COL4A1 significantly enhanced the tube formation and motility of HUVECs (Fig. 6C and D). All these effects on HUVECs induced by siTGFBI or siCOL4A1 were similar to those observed following HUVEC exposure to BCPAP cells-derived exosomes under hypoxic conditions, which further indicates that the proangiogenic effects following HUVEC exposure to BCPAPderived exosomes under hypoxic conditions are mediated by miR-21-5p-induced inhibition of TGFBI and COL4A1.

\section{Elevated exosomal miR-21-5p is found in PTC patients' sera}

To further elucidate the nature of the exosomal miR-21-5p present in the sera of PTC patients, we next compared exosomal miR-21-5p levels in sera among healthy donors $(n=20)$ and PTC patients with lymph node metastasis $(n=20)$. Circulating exosomes and miR-21-5p levels from the sera were measured by qRT-PCR (Fig. 7A). Compared with healthy donors, miR-21-5p levels were significantly higher in PTC patients. HUVECs were used to assess the pro-angiogenic activity of exosomes isolated from the sera of healthy donors and PTC patients. As shown in Fig. 7B and $\mathrm{C}$, the tube formation of HUVECs was dramatically enhanced in the presence of exosomes isolated from the patients' sera. Exosomes presenting high levels of miR-21-5p exhibited greater enhancement of tube formation. Similarly, the promotive effect of the patient' sera-derived exosomes on the enhancement of HUVECs migration was observed (Fig. 7D and E). Finally, immunohistochemical staining for TGFBI and COL4A1 revealed decreased expression of TGFBI and COL4A1 in the endothelial cells in the resection specimens from PTC patients in comparison to normal thyroid tissue from non-cancerous thyroid specimens (Fig. 7F, G and H). Our results convincingly proved that high levels of circulating miR-21-5p effectively increase angiogenesis among PTC patients.

\section{Discussion}

In this study, we provided evidence that exosomal miR-21-5p secreted by hypoxic PTC cells is a potent
A

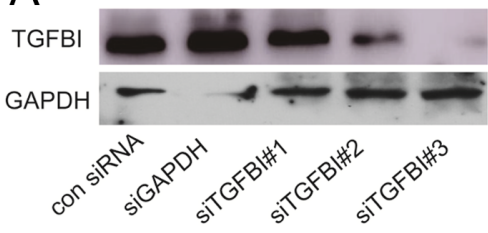

B

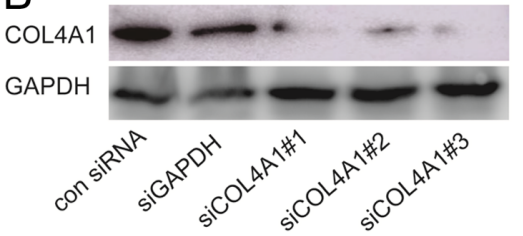

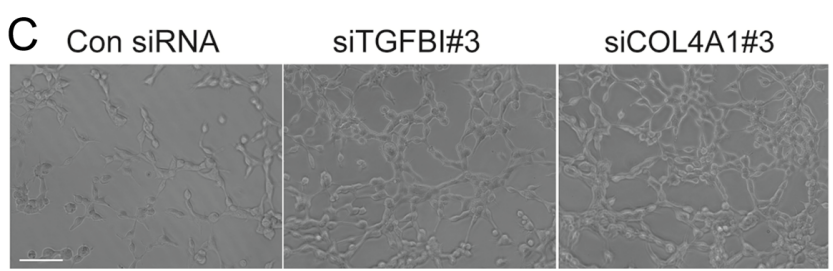

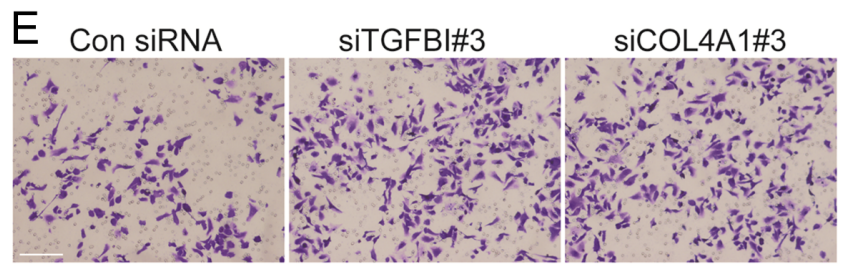

https://erc.bioscientifica.com https://doi.org/10.1530/ERC-19-0008
(C) 2019 Society for Endocrinology Published by Bioscientifica Ltd. Printed in Great Britain
D

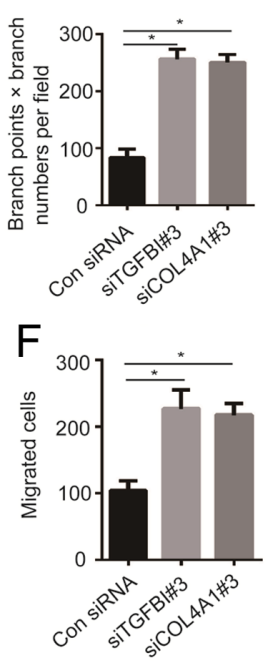

Figure 6

Inhibition of TGFBI or COL4A1 induces proangiogenic effects on endothelial cells. (A) HUVECs were transfected with control siRNA, siGAPDH or three different TGFBI siRNAs for $48 \mathrm{~h}$, and then the inhibitory efficiency of the siRNAs targeting TGFBI was verified by Western blotting; siGAPDH was used as a positive control. (B) HUVECs were transfected with control siRNA, siGAPDH or three different COL4A1 siRNAs for $48 \mathrm{~h}$, and then the inhibitory efficiency of the siRNAs targeting COL4A1 was verified by Western blotting. (C) The tube formation of HUVECs in different treatment groups was tested at $6 \mathrm{~h}$. (D) Quantitative analysis of the total branching points number in (C). $n=3$ per group. (E) The migration of HUVECs in

different treatment groups was tested at $24 \mathrm{~h}$ by the transwell migration assay. (F) Quantitative analysis of the migrated cells number in (E). $n=3$ per group. ${ }^{*} P<0.05$ compared with the control (con) siRNA group. Bars $=200 \mu \mathrm{m}$. 

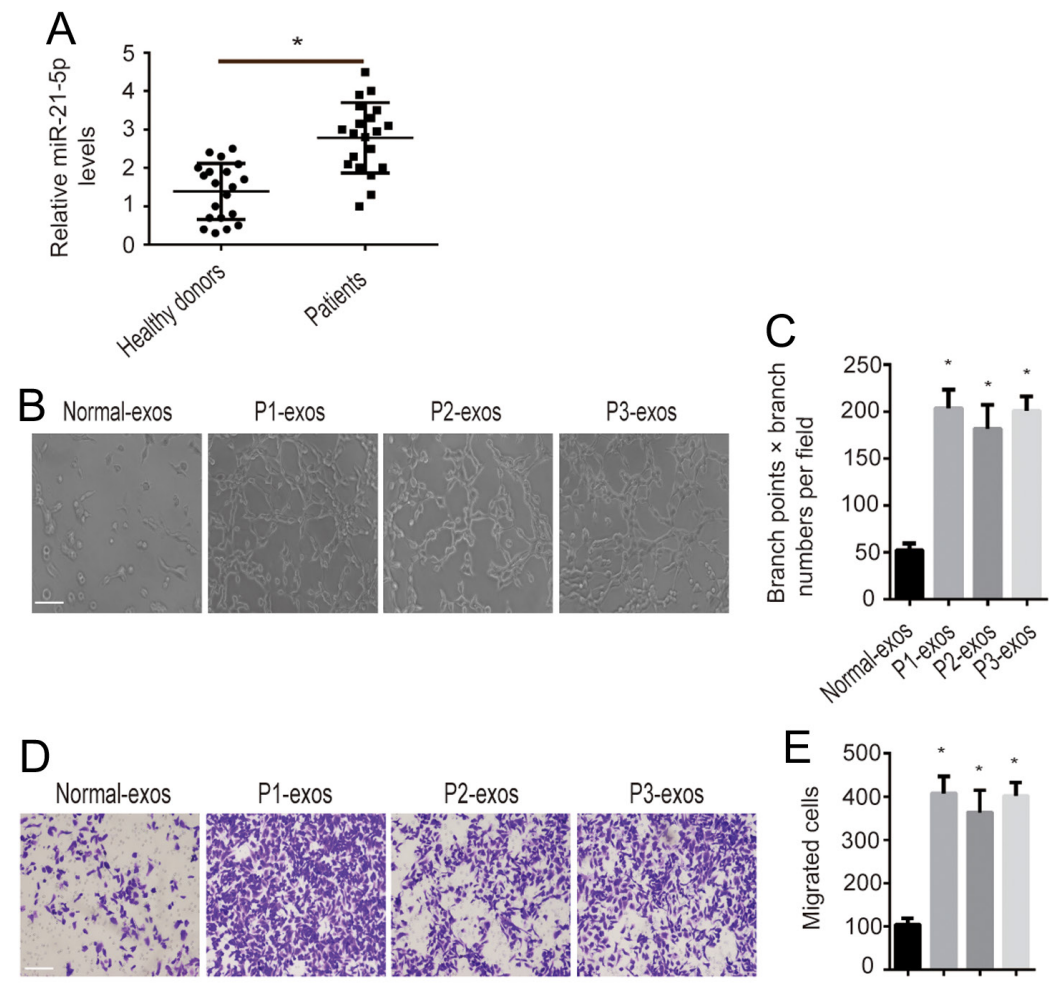
Figure 7
Elevated circulating miR-21-5p in papillary thyroid cancer patients. (A) The levels of exosomal miR-21-5p in healthy donors and papillary thyroid cancer patients $(n=20)$. (B and D) Exosomes isolated from papillary thyroid cancer patient sera enhanced the tube formation after treatment with exosomes for $6 \mathrm{~h}(\mathrm{~B})$ and migration after treatment with exosomes for $24 \mathrm{~h}$ (D). (C) Quantitative analysis of the total branching points and total tube length in (B). $n=3$ per group. (E) Quantitative analysis of the migration rates in (D). $n=3$ per group. Each value is the mean \pm S.D. of three determinations. (F) Immunohistochemical staining with anti-human TGFBI or COL4A1 antibody between the normal thyroid tissues and papillary thyroid cancer tissues. $(\mathrm{G}, \mathrm{H}) \mathrm{IHC}$ score in (F) were analysed. $* P<0.05$ compared with the control (con) siRNA group. Bars $=200 \mu \mathrm{m}$.

pro-angiogenic factor through its targeting of TGFBI and COL4A1 both in vitro and in vivo (Fig. 8). The crosstalk between cancer cells and endothelial cells by exosomal miR-21-5p in the TME studied here confirms that miR-21-5p may be a predictive biomarker or a potential strategy for PTC treatment.

In recent years, emerging evidence implicates the TME as a contributor to cancer development (Rankin \& Giaccia 2016, Trikha et al. 2016). Exosomes represent important mediators of cancer-TME communication, both locally and at distant sites (Hosseini-Beheshti et al. 2016). Exosomes have been implicated as playing critical roles in almost all aspects of oncology, such as tumourigenesis, proliferation, apoptosis, invasion, metastasis and chemoresistance, by regulating the expression of various oncogenes or tumour suppressor genes by transferring miRNAs to recipient cells (Cui et al. 2015, Kohlhapp et al. 2015). Cancer cells increase tumour angiogenesis and cancer metastasis by releasing exosomes enriched with miR-351b and miR-210 (Kosaka et al. 2013). Hypoxic lung cancer cell-derived exosomal miR-23a not only increased tumour angiogenesis during hypoxia but also increased it in normoxic conditions (Hsu et al. 2017). In this study, we found a novel mechanism involving thyroid cancer pathogenesis, and our result showed that exosomal miR-21-5p isolated from hypoxic thyroid cancer cells increased tumour angiogenesis and inhibition of miR-21-5p reduced angiogenesis both in vitro and in vivo, 


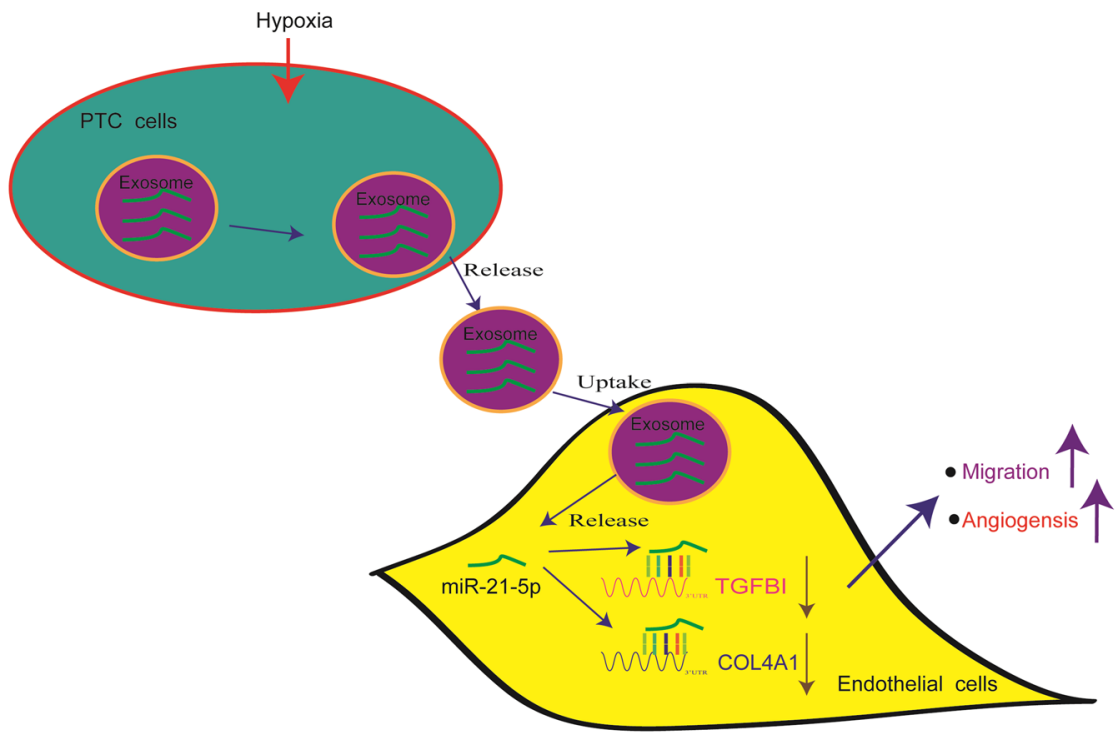

\section{Figure 8}

A proposed model of hypoxic papillary thyroid cancer cells increase angiogenesis via exosomal miRNA. Exosomal miR-21-5p secreted by hypoxic PTC cells is a potent pro-angiogenic factor through its targeting of TGFBI and COL4A1 both in vitro and in vivo. which suggested that hypoxic thyroid cancer transmits genetic material between cancer cells and endothelial cells, regulating angiogenesis in the TME. Moreover, since circulating miR-21-5p levels are increased in the plasma of patients with PTC with lymph node metastasis, this suggests that exosomal miR-21-5p might be one of the key factors for tumour angiogenesis.

miRNAs are a class of 20-24nt short non-coding RNAs that post-transcriptionally regulate gene expression (Au Yeung et al. 2016). miR-21-5p is one of the most wellstudied miRNAs and is implicated in promoting cancer growth and development that is often overexpressed in a wide variety of solid tumour tissues (Yao et al. 2009). miR-21's pro-angiogenic function has been previously described and involves a synergistic effect with VEGF. In addition, miR-21-5p has been found to increase angiogenesis by targeting multiple signalling pathways in various cancers, such as prostate cancer (Liu et al. 2011) and lung cancer (Zhao et al. 2013). However, there is little information about whether miR-21-5p is involved in the pro-angiogenic activity of PTC-derived exosomes. In this work, we are the first to demonstrate that PTCderived exosomal miR-21-5p can promote angiogenesis both in vitro and in vivo. Moreover, inhibition of exosomal miR-21-5p in PTC cells resulted in attenuating exosomemediated angiogenesis both in vitro and in vivo. Thus, therapies targeting miR-21-5p and its target protein factors, in combination with existing conventional therapies, may provide us an effective treatment for cancer patients with a high risk of metastasis.

Nthy-ori-3-1 is a normal thyroid cell line, which had been widely used in thyroid researches (Li et al. 2012).
Here, we detected high levels of miR-21-5p, but not miR-21-3p, expressed in hypoxic BCPAP cell-derived exosomes, but not in normal thyroid Nthy-ori-3-1 cells or normoxic BCPAP cell-derived exosomes. After incubating with hypoxic BCPAP cell-derived exosomes for $3 \mathrm{~h}$, we found that miR-21-5p expression was remarkably enhanced in HUVECs, indicating that miR-21-5p can be transferred from hypoxic BCPAP cell-derived exosomes to recipient cells. Studies have demonstrated that TGFBI and COL4A1 are anti-angiogenic factors (Skonier et al. 1992, Zhao et al. 2002, 2006, Kalluri 2003, Nam et al. 2005, Shao et al. 2006, Khoshnoodi et al. 2008, Zhang et al. 2009, Assadian et al. 2012). In the present study, we identified both TGFBI and COL4A1 as the targets of miR-21-5p. Moreover, knockdown of TGFBI and COL4A1 could result in attenuating the pro-angiogenic effects of exosomes from hypoxic PTC cells. We found in endothelial cells that ectopically cancer-derived miR-21-5p transferred via exosomes can effectively reduce TGFBI and COL4A1 expression and reduce tube formation.

At last, we found that exosomal miR-21-5p levels were significantly upregulated in PTC sera specimens. Also, these PTC patient-derived exosomes dramatically enhanced tube formation and migration of HUVECs. More importantly, low expression of TGFBI and COL4A1 were detected in the endothelial cells in the resection specimens from PTC patients in comparison to normal thyroid tissue from non-cancerous thyroid specimens. These clinical data imply that the miR-21-5p/TGFBI/COL4A1 axis may play roles in the PTC angiogenesis in vivo.
C) 2019 Society for Endocrinology Published by Bioscientifica Ltd. Printed in Great Britain 
Taken together, our study revealed the cell interactions between hypoxic cancer cells and endothelial cells, elucidating a new mechanism by which hypoxic PTC cells increase angiogenesis via a previously undescribed exosomal miR-21-5p/TGFBI and miR-21-5p/COL4A1 regulatory pathway. This essential communication by secretory exosomes, which connects hypoxic cancer cells and endothelial cells, may provide novel options for preventive therapies in the future and may have functional consequences in developing personalised diagnostics and therapeutics for PTC patients.

\section{Supplementary data}

This is linked to the online version of the paper at https://doi.org/10.1530/ ERC-19-0008.

\section{Declaration of interest}

The authors declare that there is no conflict of interest that could be perceived as prejudicing the impartiality of the research reported.

\section{Funding}

This work was supported by funding from the National Natural Science Foundation of China (No. 81770881 and 81870623), and the New Xiang-Ya Talent Project of the Third Xiang-Ya Hospital of Central South University.

\section{References}

Akslen LA \& Livolsi VA 2000 Increased angiogenesis in papillary thyroid carcinoma but lack of prognostic importance. Human Pathology $\mathbf{3 1}$ 439-442. (https://doi.org/10.1053/1-ip.2000.6548)

Aschebrook-Kilfoy B, Kaplan EL, Chiu BC, Angelos P \& Grogan RH 2013 The acceleration in papillary thyroid cancer incidence rates is similar among racial and ethnic groups in the United States. Annals of Surgical Oncology 20 2746-2753. (https://doi.org/10.1245/s10434-013-2892-y)

Assadian S, El-Assaad W, Wang XQ, Gannon PO, Barres V, Latour M, Mes-Masson AM, Saad F, Sado Y, Dostie J, et al. 2012 p53 inhibits angiogenesis by inducing the production of Arresten. Cancer Research 72 1270-1279. (https://doi.org/10.1158/0008-5472.CAN-11-2348)

Au Yeung CL, Co NN, Tsuruga T, Yeung TL, Kwan SY, Leung CS, Li Y, Lu ES, Kwan K, Wong KK, et al. 2016 Exosomal transfer of stromaderived miR21 confers paclitaxel resistance in ovarian cancer cells through targeting APAF1. Nature Communications 7 11150. (https:// doi.org/10.1038/ncomms11150)

Bayraktar R, Ivan C, Bayraktar E, Kanlikilicer P, Kabil NN, Kahraman N, Mokhlis HA, Karakas D, Rodriguez-Aguayo C, Arslan A, et al. 2018 Dual suppressive effect of miR-34a on the FOXM1/eEF2-kinase axis regulates triple-negative breast cancer growth and invasion. Clinical Cancer Research 24 4225-4241. (https://doi.org/10.1158/1078-0432. CCR-17-1959)

Chen CY, Rao SS, Ren L, Hu XK, Tan YJ, Hu Y, Luo J, Liu YW, Yin H, Huang J, et al. 2018 Exosomal DMBT1 from human urine-derived stem cells facilitates diabetic wound repair by promoting angiogenesis. Theranostics 8 1607-1623. (https://doi.org/10.7150/ thno.22958)
Cui H, Seubert B, Stahl E, Dietz H, Reuning U, Moreno-Leon L, Ilie M, Hofman P, Nagase H, Mari B, et al. 2015 Tissue inhibitor of metalloproteinases-1 induces a pro-tumourigenic increase of miR-210 in lung adenocarcinoma cells and their exosomes. Oncogene $\mathbf{3 4}$ 3640-3650. (https://doi.org/10.1038/onc.2014.300)

Cui RR, Li SJ, Liu LJ, Yi L, Liang QH, Zhu X, Liu GY, Liu Y, Wu SS, Liao XB, et al. 2012 MicroRNA-204 regulates vascular smooth muscle cell calcification in vitro and in vivo. Cardiovascular Research 96 320-329. (https://doi.org/10.1093/cvr/cvs258)

Fagin JA \& Wells SA Jr 2016 Biologic and clinical perspectives on thyroid cancer. New England Journal of Medicine 375 1054-1067. (https://doi.org/10.1056/NEJMra1501993)

Hosseini-Beheshti E, Choi W, Weiswald LB, Kharmate G, Ghaffari M, Roshan-Moniri M, Hassona MD, Chan L, Chin MY, Tai IT, et al. 2016 Exosomes confer pro-survival signals to alter the phenotype of prostate cells in their surrounding environment. Oncotarget 7 14639-14658. (https://doi.org/10.18632/oncotarget.7052)

Hsu YL, Hung JY, Chang WA, Lin YS, Pan YC, Tsai PH, Wu CY \& Kuo PL 2017 Hypoxic lung cancer-secreted exosomal miR-23a increased angiogenesis and vascular permeability by targeting prolyl hydroxylase and tight junction protein ZO-1. Oncogene $\mathbf{3 6}$ 4929-4942. (https://doi.org/10.1038/onc.2017.105)

Jemal A, Siegel R, Ward E, Hao Y, Xu J, Murray T \& Thun MJ 2008 Cancer statistics, 2008. CA: A Cancer Journal for Clinicians 58 71-96. (https://doi.org/10.3322/CA.2007.0010)

Kalluri R 2003 Basement membranes: structure, assembly and role in tumour angiogenesis. Nature Reviews Cancer 3 422-433. (https://doi. org/10.1038/nrc1094)

Khoshnoodi J, Pedchenko V \& Hudson BG 2008 Mammalian collagen IV. Microscopy Research and Technique 71 357-370. (https://doi. org/10.1002/jemt.20564)

Kohlhapp FJ, Mitra AK, Lengyel E \& Peter ME 2015 MicroRNAs as mediators and communicators between cancer cells and the tumor microenvironment. Oncogene 34 5857-5868. (https://doi. org/10.1038/onc.2015.89)

Kong W, He L, Richards EJ, Challa S, Xu CX, Permuth-Wey J, Lancaster JM, Coppola D, Sellers TA, Djeu JY, et al. 2014 Upregulation of miRNA-155 promotes tumour angiogenesis by targeting VHL and is associated with poor prognosis and triplenegative breast cancer. Oncogene 33 679-689. (https://doi. org/10.1038/onc.2012.636)

Kosaka N, Iguchi H, Hagiwara K, Yoshioka Y, Takeshita F \& Ochiya T 2013 Neutral sphingomyelinase 2 (nSMase2)-dependent exosomal transfer of angiogenic microRNAs regulate cancer cell metastasis. Journal of Biological Chemistry 288 10849-10859. (https://doi. org/10.1074/jbc.M112.446831)

Lee JC, Gundara JS, Glover A, Serpell J \& Sidhu SB 2014 MicroRNA expression profiles in the management of papillary thyroid cancer. Oncologist 19 1141-1147. (https://doi.org/10.1634/ theoncologist.2014-0135)

Li X, Wang Z, Liu J, Tang C, Duan C \& Li C 2012 Proteomic analysis of differentially expressed proteins in normal human thyroid cells transfected with PPFP. Endocrine-Related Cancer 19 681-694. (https:// doi.org/10.1530/ERC-12-0156)

Liao XB, Zhang ZY, Yuan K, Liu Y, Feng X, Cui RR, Hu YR, Yuan ZS, Gu L, Li SJ, et al. 2013 MiR-133a modulates osteogenic differentiation of vascular smooth muscle cells. Endocrinology 154 3344-3352. (https://doi.org/10.1210/en.2012-2236)

Lim H, Devesa SS, Sosa JA, Check D \& Kitahara CM 2017 Trends in thyroid cancer incidence and mortality in the United States, 1974-2013. JAMA 317 1338-1348. (https://doi.org/10.1001/jama.2017.2719)

Lin X, Xu F, Cui RR, Xiong D, Zhong JY, Zhu T, Li F, Wu F, Xie XB, Mao MZ, et al. 2018 Arterial calcification is regulated via an miR204/DNMT3a regulatory circuit both in vitro and in female mice. Endocrinology 159 2905-2916. (https://doi.org/10.1210/en.201800320) 
Liu L, Li C, Chen Q, Jing Y, Carpenter R, Jiang Y, Kung H, Lai L, Jiang B, et al. 2011 MiR-21 induced angiogenesis through AKT and ERK activation and HIF-1 $\alpha$ expression. PLOS ONE 6 e19139. (https://doi. org/10.1371/journal.pone.0019139)

Liu Y, Luo F, Wang B, Li H, Xu Y, Liu X, Shi L, Lu X, Xu W, Lu L, et al. 2016a STAT3-regulated exosomal miR-21 promotes angiogenesis and is involved in neoplastic processes of transformed human bronchial epithelial cells. Cancer Letters 370 125-135. (https://doi. org/10.1016/j.canlet.2015.10.011)

Liu Y, Xu F, Pei HX, Zhu X, Lin X, Song CY, Liang QH, Liao EY \& Yuan LQ 2016 $b$ Vaspin regulates the osteogenic differentiation of MC3T3-E1 through the PI3K-Akt/miR-34c loop. Scientific Reports 6 25578. (https://doi.org/10.1038/srep25578)

Nam JO, Jeong HW, Lee BH, Park RW \& Kim IS 2005 Regulation of tumor angiogenesis by fastatin, the fourth FAS1 domain of betaig-h3, via alphavbeta3 integrin. Cancer Research 65 4153-4161. (https://doi.org/10.1158/0008-5472.CAN-04-2705)

Pientka FK, Hu J, Schindler SG, Brix B, Thiel A, Johren O, Fandrey J, Berchner-Pfannschmidt U \& Depping R 2012 Oxygen sensing by the prolyl-4-hydroxylase PHD2 within the nuclear compartment and the influence of compartmentalisation on HIF-1 signalling. Journal of Cell Science 125 5168-5176. (https://doi.org/10.1242/jcs.109041)

Quail DF \& Joyce JA 2013 Microenvironmental regulation of tumor progression and metastasis. Nature Medicine 19 1423-1437. (https:// doi.org/10.1038/nm.3394)

Rankin EB \& Giaccia AJ 2016 Hypoxic control of metastasis. Science 352 175-180. (https://doi.org/10.1126/science.aaf4405)

Shao G, Berenguer J, Borczuk AC, Powell CA, Hei TK \& Zhao Y 2006 Epigenetic inactivation of Betaig-h3 gene in human cancer cells. Cancer Research 66 4566-4573. (https://doi.org/10.1158/0008-5472. CAN-05-2130)

Skonier J, Neubauer M, Madisen L, Bennett K, Plowman GD \& Purchio AF 1992 cDNA cloning and sequence analysis of beta ig-h3, a novel gene induced in a human adenocarcinoma cell line after treatment with transforming growth factor-beta. DNA and Cell Biology 11 511-522. (https://doi.org/10.1089/dna.1992.11.511)

Sun W, Wang X, Li J, You C, Lu P, Feng H, Kong Y, Zhang H, Liu Y, Jiao R, et al. 2018 MicroRNA-181a promotes angiogenesis in colorectal cancer by targeting SRCIN1 to promote the SRC/VEGF signaling pathway. Cell Death and Disease 9 438. (https://doi. org/10.1038/s41419-018-0490-4)

Tadokoro H, Umezu T, Ohyashiki K, Hirano T \& Ohyashiki JH 2013 Exosomes derived from hypoxic leukemia cells enhance tube formation in endothelial cells. Journal of Biological Chemistry 288 34343-34351. (https://doi.org/10.1074/jbc.M113.480822)

Tonini T, Rossi F \& Claudio PP 2003 Molecular basis of angiogenesis and cancer. Oncogene 22 6549-6556. (https://doi.org/10.1038/sj. onc.1206816)
Trikha P, Sharma N, Pena C, Reyes A, Pecot T, Khurshid S, Rawahneh M, Moffitt J, Stephens JA, Fernandez SA, et al. 2016 E2F3 in tumor macrophages promotes lung metastasis. Oncogene 35 3636-3646. (https://doi.org/10.1038/onc.2015.429)

Triner D \& Shah YM 2016 Hypoxia-inducible factors: a central link between inflammation and cancer. Journal of Clinical Investigation 126 3689-3698. (https://doi.org/10.1172/JCI84430)

Wang W, Wen Q, Luo J, Chu S, Chen L, Xu L, Zang H, Alnemah MM, Li J, Zhou J, et al. 2017 Suppression of beta-catenin nuclear translocation by CGP57380 decelerates poor progression and potentiates radiation-induced apoptosis in nasopharyngeal carcinoma. Theranostics 7 2134-2149. (https://doi.org/10.7150/thno.17665)

Wu YC, Tang SJ, Sun GH \& Sun KH 2016 CXCR7 mediates TGFbeta1promoted EMT and tumor-initiating features in lung cancer. Oncogene 35 2123-2132. (https://doi.org/10.1038/onc.2015.274)

Yang F, Wang W, Zhou C, Xi W, Yuan L, Chen X, Li Y, Yang A, Zhang J \& Wang T 2015 MiR-221/222 promote human glioma cell invasion and angiogenesis by targeting TIMP2. Tumour Biology 36 3763-3773. (https://doi.org/10.1007/s13277-014-3017-3)

Yao Q, Xu H, Zhang QQ, Zhou H \& Qu LH 2009 MicroRNA-21 promotes cell proliferation and down-regulates the expression of programmed cell death 4 (PDCD4) in HeLa cervical carcinoma cells. Biochemical and Biophysical Research Communications 388 539-542. (https://doi. org/10.1016/j.bbrc.2009.08.044)

Yuan LQ, Liu W, Cui RR, Wang D, Meng JC, Xie H, Wu XP, Zhou HD, Lu Y \& Liao EY 2010 Taurine inhibits osteoclastogenesis through the taurine transporter. Amino Acids 39 89-99. (https://doi.org/10.1007/ s00726-009-0380-2)

Zhang Y, Wen G, Shao G, Wang C, Lin C, Fang H, Balajee AS, Bhagat G, Hei TK \& Zhao Y 2009 TGFBI deficiency predisposes mice to spontaneous tumor development. Cancer Research 69 37-44. (https:// doi.org/10.1158/0008-5472.CAN-08-1648)

Zhao Y, El-Gabry M \& Hei TK 2006 Loss of Betaig-h3 protein is frequent in primary lung carcinoma and related to tumorigenic phenotype in lung cancer cells. Molecular Carcinogenesis 45 84-92. (https://doi. org/10.1002/mc.20167)

Zhao Y, Xu Y, Luo F, Xu W, Wang B, Pang Y, Zhou J, Wang X \& Liu Q 2013 Angiogenesis, mediated by miR-21, is involved arseniteinduced carcinogenesis. Toxicology Letters 223 35-41. (https://doi. $\operatorname{org} / 10.1016 / j . t o x l e t .2013 .08 .020)$

Zhao YL, Piao CQ \& Hei TK 2002 Downregulation of Betaig-h3 gene is causally linked to tumorigenic phenotype in asbestos treated immortalized human bronchial epithelial cells. Oncogene $\mathbf{2 1}$ 7471-7477. (https://doi.org/10.1038/sj.onc.1205891)

Zhou W, Fong MY, Min Y, Somlo G, Liu L, Palomares MR, Yu Y, Chow A, O'Connor ST, Chin AR, et al. 2014 Cancer-secreted miR-105 destroys vascular endothelial barriers to promote metastasis. Cancer Cell 25 501-515. (https://doi.org/10.1016/j.ccr.2014.03.007)

Received in final form 14 February 2019

Accepted 14 March 2019

Accepted Preprint published online 14 March 2019 (c) 2019 Society for Endocrinology Published by Bioscientifica Ltd. Printed in Great Britain 